\title{
Improvement Research on S-MAC Protocol in Wireless Sensor Network
}

\author{
Yan $\mathrm{Shi}^{{ }^{*}}{ }^{*}$ Jing $\mathrm{Jin}^{2}$ and Jianxian Cai ${ }^{1}$ \\ ${ }^{1}$ Department of Disaster Prevention Instrument, Institute of Disaster Prevention, \\ No.3, University Street, Yanjiao Development District, Sanhe, Hebei 065201, \\ China \\ ${ }^{2}$ Information Center, Chinese Academy of Land and Resource Economics, No.689, \\ Jingyu Street, Yanjiao Development District, Sanhe, Hebei 101149, China \\ Corresponding author email: windsor33@163.com
}

\begin{abstract}
This paper strengthens the application of adaptive listening technology in S-MAC. When the nodes have no data to process, they are in dormancy state for most of the time, while when the data is coming, they become active quickly and participate in data transmission actively. The nodes which can feel the transmission is just around will participate in adaptive transmission as much as possible and make continuous adaptive listening within one dormancy period. In this way, realize the purpose of deceasing end to end delay greatly on the basis of not increasing additional energy consumption. For applications which are sensitive to system response time, the improved S-MAC is with greater application value.
\end{abstract}

Keywords: S-MAC Protocol, Wireless Sensor Network, listening technology, improved $S-M A C$

\section{Introduction}

Energy efficiency problem is a main problem in wireless sensor network. To solve the energy efficiency problem, each process in the design of wireless sensor network needs to consider about energy consumption problem and there is also no exception in the design of MAC protocol [1]. At present, mainstream MAC protocols use regular dormancy technology basically, which is to turn off the powerconsuming radio transceiver when the node confirms not needing communication and then reach the energy saving purpose. The cost for the adoption of dormancy technology is the serious delay of system from end to end. For application field with higher requirement for real time, delay problem will become one bottleneck in wireless sensor network technology.

For this situation, we have deepened the application of adaptive listening technology in S-MAC based on S-MAC protocol. When the nodes have no data to process, most of them are in formant state, while when the data is coming, they become very active and participate in data transmission actively. Those nodes feeling that data transmission is just around will participate in adaptive transmission as far as possible, while within one dormant period, these nodes make adaptive listening continuously [2]. To decrease the energy consumption of the system, we will also make other improvements, such as decreasing the nodes number of invalid adaptive listening, and then realize the purpose of decreasing end to end delay greatly on the basis of not increasing system additional energy consumption.

In this paper, S-MAC without adoption of adaptive listening technology is called as basic S-MAC and S-MAC with adoption of adaptive listening technology is called as 
adaptive S-MAC, S-MAC after modification is called as improved S-MAC. S-MAC not specially specified usually refers to basic S-MAC and adaptive S-MAC [3].

\section{S-MAC Principle}

Before making specific description for S-MAC components, firstly, introduce wireless sensor network and imagined scene of application briefly. Wireless sensor network is composed of a lot of nodes making use of short distance and multi-hop communications to reach the purpose of energy saving [4]. Most of communications are peer to peer communications among nodes but not communication between node and one single base station. In-network processing is of great importance for network lifetime, which means that under the mode of store-forward, the data will be processed. Grouping or multiple-source fragmentation only increases the delay of the whole network.

\subsection{Periodic Monitoring and Dormancy}

S-MAC decreases listening time through making periodic dormancy for nodes and the basic program is as shown in Figure 1. Each node will wake up after dormancy for some time, and then listening, sending and receiving data as well as other control grouping can be made at this moment [5]. Nodes can know if there are other nodes need to communicate with them through listening at this moment. If it wants to communicate with other nodes actively, it should contact other nodes during this time. During dormancy, nodes turn down radio transceiver and set the timer to wake them up.

All the nodes can select their own listening/dormancy timetable freely, however, to decrease and save expenses, S-MAC is more likely to make mutual synchronization among neighbouring nodes [6]. In this way, they are listening at the same time and dormant at the same time. It needs to pay attention that in one multi-hop network, not all the neighbouring nodes can be synchronized together [7]. As shown in Figure 2, if nodes A, D synchronize with nodes $\mathrm{C}, \mathrm{D}$, and then neighbouring nodes A and B may have different timetables.

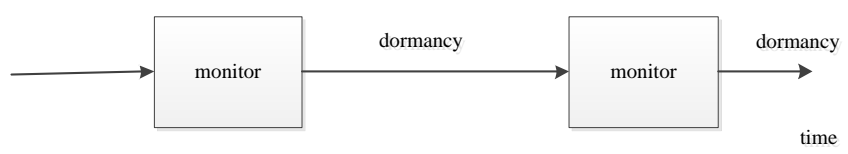

Figure 1. Periodic Monitoring and Dormancy

\subsection{Avoid Conflicts}

If there are several neighbours wanting to make dialogue with one node at the same time, they will deliver as the listening starts. Under this situation, all of them need to compete for media [8-9]. In competition agreement, 802.11 deals better in avoiding conflicts. S-MAC protocol adopts the same processing, including virtual and physical carrier sense as well as RTS/CTS exchange for problems at hidden end. In the grouping of each transmission, there is one continuous domain to indicate how long will this transmission lasts [10-12]. If one node receives a grouping that should be sent to other nodes, it will know how long it shall keep in silence from this domain. The node records the value into one variable named network allocation vector (NAV) and then set one timer for it. Every time when the timer starts up, node decreases NAV value until it becomes zero. 


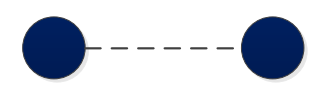

$\mathrm{C}$

A

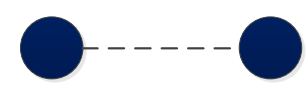

B

D

\section{Figure 2. Adjacent Nodes A and B have different Schedules, and C and D Respectively}

\subsection{Coordinate dormancy}

For idle listening, periodic dormancy decreases the waste of energy effectively. In S-MAC, node adjusts their dormancy time but not their random dormancy [13]. The following describes the process of all nodes following setting and maintaining their timetable as well as the adopted adaptive listening technology to decrease dormancy delay.

Figure 3 displays the time relationship of three possible situations of sender transmitting to receiver. Sender 1 only sends out SNYC grouping, sender 2 only sends out one unicast data packet and sender 3 sends out both.

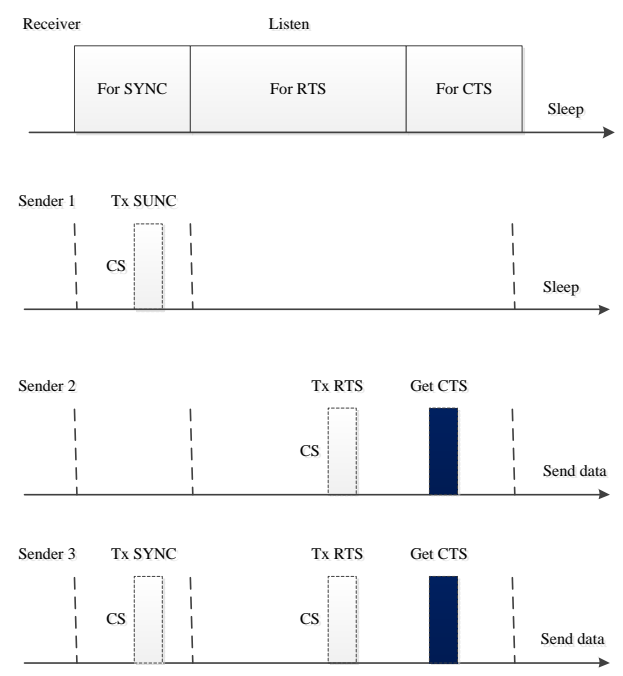

Figure 3. The Sender and the Receiver of Communication Three Possible Situations

\section{S-MAC Improvement and Delay Analysis}

\subsection{Increase Adaptive Transmission Hop}

Based on the idea of adaptive listening, it must let more nodes listen to the existence of data transmission to increase the times of data transmission in adaptive listening and then increase the number of nodes which wake up in advance [14]. Therefore, it needs to make research on node communication distance first. For the theory of wireless communication as well as the mature theory for measurement, it mainly presents in the following formula:

$$
P_{r} \propto P_{t} d^{\beta}
$$

In the above formula, Pr is the energy of receiving signal, pt is the energy of transmitting signal, $\mathrm{d}$ is signal transmission distance, $\beta$ is constant depending on environment; based on the differences of transmission medium, its value is between 2 and 5[15]. A conclusion can be drawn from above formula that the energy of receiving signal Pr decreases with the increasing of transmission distance d. It can be seen obviously that 
within certain distance, transmission signal will not stop suddenly with the increasing of distance but weaken gradually until the signal disappears [16-17]. In Figure 4, node B can receive the RTS grouping sent by node A correctly, while the possibility of correct receiving of node $\mathrm{C}$ decreases greatly. It can't be received correctly, but node $\mathrm{C}$ can receive weak signal, which is to say that it can also listen to the transmission of data, which will be explained in the following. The transmission at this time is used to agree the listening cycle of data transmission, therefore, node $\mathrm{C}$ can de deduced, which is the handshake of neighboring nodes for data transmission during dormancy. For node $\mathrm{C}$ (even D and E) listening to data transmission, it can be added into adaptive listening activity during dormancy. In this way, it can increase the quantity of adaptive transmission nodes, at the same time, increase adaptive transmission distance and the delay of the whole application system will be decreased accordingly.

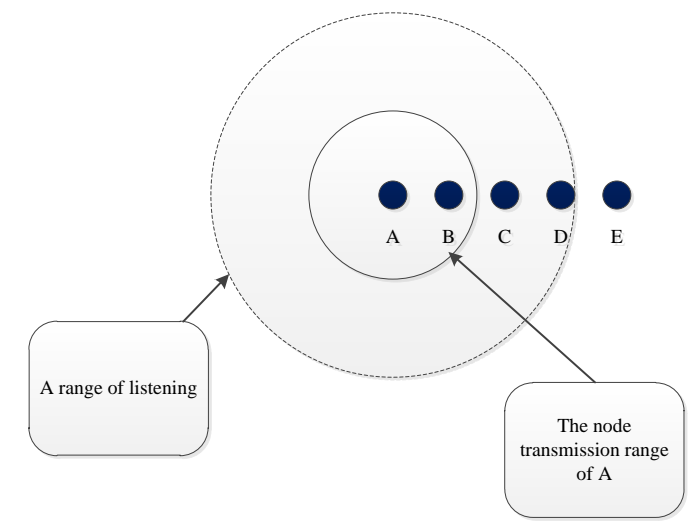

Figure 4. Transmission Range is usually Listen Twice

There is also one question in above analysis, how far the signal is transmitted so that the node cannot listen? It can be learnt that the carrier sensing range is about two times of transmission range, as shown in Figure 4.

Different from adaptive S-MAC, our improvement is to wake up the nodes listening to RTS and CTS in advance, and then participate in several adaptive transmissions going to start. This process can be indicated.

First, A, B nodes exchange RTS and CTS at listening cycle and all the nodes within the listening range of these two nodes can be listened. During the dormancy period after listening period, except nodes $\mathrm{A}$ and $\mathrm{B}$, all the other nodes enter into dormancy state. Nodes A and B make data transmission, as shown in Figure 5.

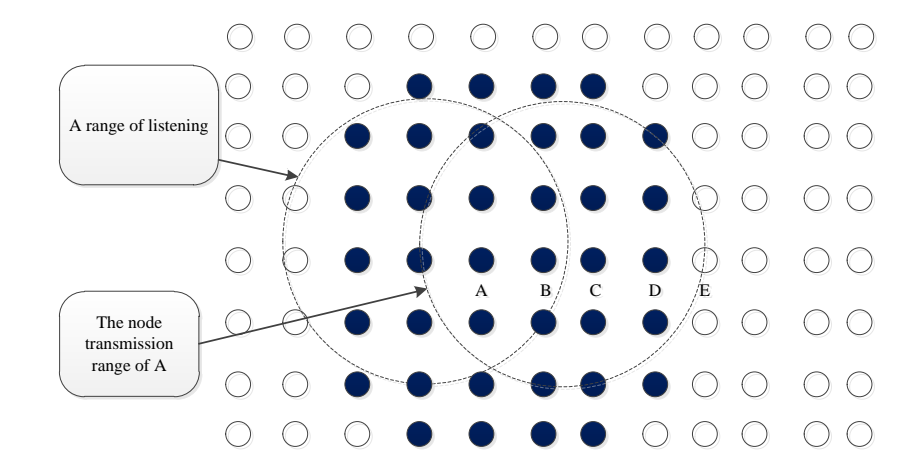

Figure 5. Node A and B for Adaptive Transmission

At the end of nodes, A and B making data transmission, all the nodes participating in adaptive transmission wake up. B and C make RTS and CTS exchange; all the other 
wakened nodes make listening, within a period of time, nodes not being listened enter into dormancy state [18-22]. All the other nodes enter dormancy state after listening to the CTS of node C and wake up after a period of dormancy (for nodes receiving RTS or CTS grouping, it is to control the time length indicated in NAV domain of RTS grouping or CTS grouping, or it is the transmission average time length of data grouping, the same below), as shown in Figure 6.

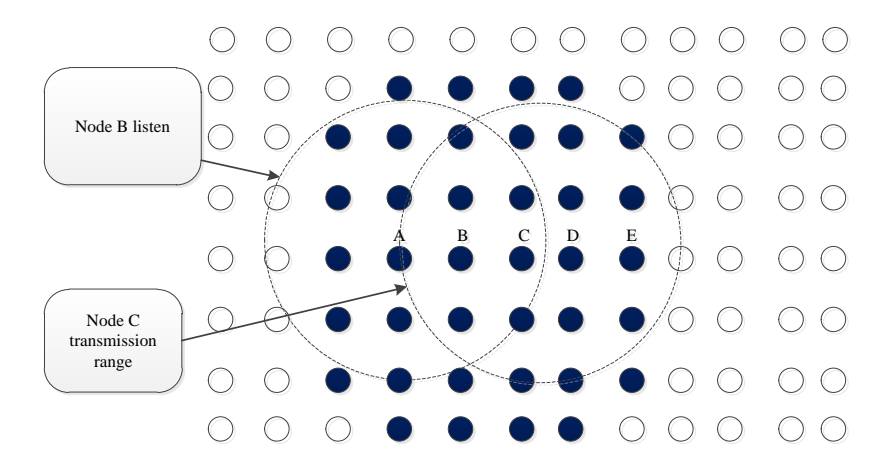

Figure 6. Node B and C for Adaptive Transmission

After nodes B and C making data exchange, their adaptive nodes wake up. C and D exchange RTS and CTS, other wakened nodes make listening. Within a period of time, nodes not listened enter dormancy state. Other nodes enter dormancy state after listening to the CTS of node D and wake up again after sleeping for a fixed time, as shown in Figure 7.

After nodes $\mathrm{C}$ and $\mathrm{D}$ making data exchange, their adaptive nodes wake up. D sends out RTS and other wakened nodes make listening. Within a certain period of time, there is no node reflecting to the RTS of D, and then all the nodes enter dormancy state, as shown in Figure 8.

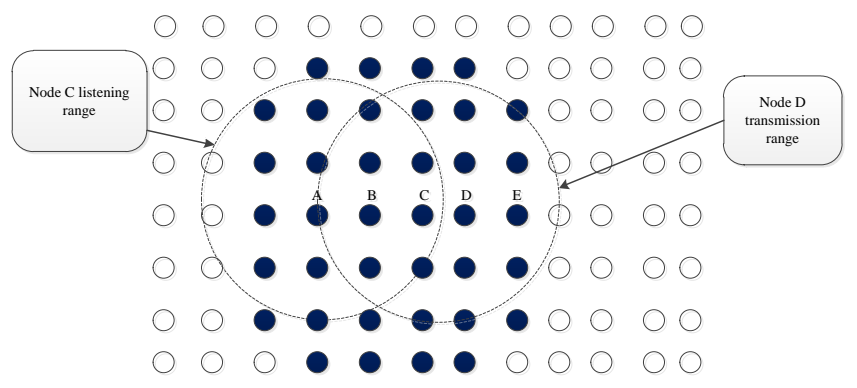

Figure 7. C and D node of Adaptive Transmission

After an average data transmission time, adaptive nodes wake up; wait for a fixed time and all will enter dormancy state if no control grouping is listened.

In this way, realize adaptive continuous transmission with various nodes participation within one dormancy period and decrease the delay of system transmission. 


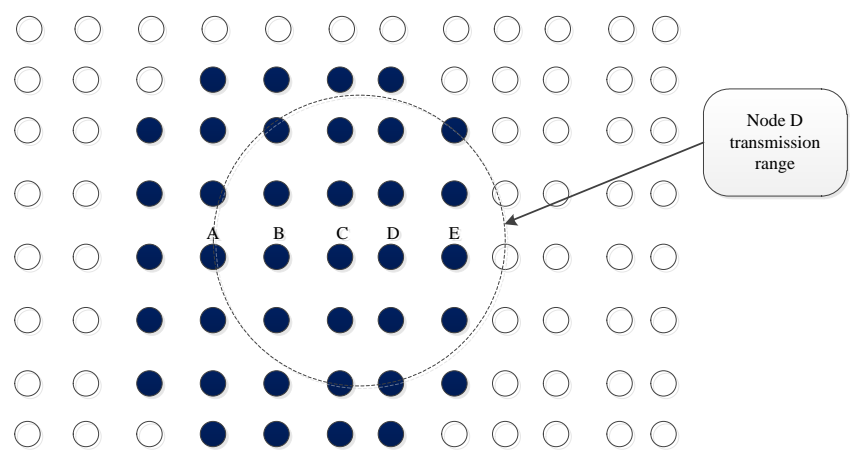

Figure 8. Nodes D cannot suit with Dormant Node E for Transmission

\subsection{Decrease Useless Adaptive Listening Node}

To decrease this cost, we have made improvement as shown in Figure 9. In the listening process, except for next hop node, all the other nodes can receive RTS sent out by node A do not need to participate in the adaptive transmission going to start. This improvement principle is very simple, under a multi-hop data transmission environment, if data reaches node $\mathrm{B}$ and next hop node $\mathrm{C}$ is within the transmission range of node $\mathrm{A}$, and then there is no need to transmit node $\mathrm{A}$ to node $\mathrm{C}$ through node B. Therefore, except for node B, node A transmits all nodes within the range (nodes within slash area), it will not become the next node in the adaptive transmission process going to start, which is to say that they do not need to wake up to waste the energy. It can decrease the number of useless nodes wake up in advance to save the energy after improvement. During dormancy as well as following multiple adaptive transmission process, this improvement is still effective.

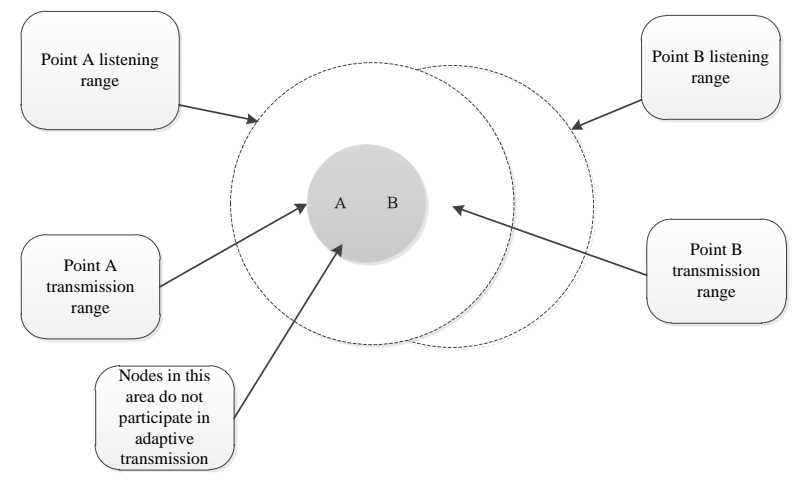

\section{Figure 9. In Addition to the Node B, to Receive A node RTS Nodes do not have to attend the Adaptive Transmission}

\subsection{Shorten adaptive listening time}

The first part is used for SYNC grouping and the second part is used to agree the RTS and CTS grouping of transmission data grouping; we call this listening as normal listening, corresponding to this is the listening in adaptive transmission and we name it as adaptive monitoring, as shown in Figure 10. During normal listening, as it needs to meet the contention of multiple nodes for shared medium, the success rate is not very high, so the listening time should be long to meet the needs of shared channel of various nodes; it is not the same in adaptive transmission process[21, 22]. When the listening starts, only nodes that have data to send use medium to send out RTS, for other nodes listening, there is no medium competition. In the following, after the next node receiving RTS, all the other nodes will be in listening state (including nodes have data to send out), therefore, there is not 
medium competition. It can be seen that the success rate of carrier sense is very high, so the RTS/CTS handshake process can be accomplished successfully in a short time. Thus, in the listening process of adaptive transmission, it is much shorter than normal listening process. This design not only decreases the listening time participating in adaptive listening node, but also decreases excessive energy consumption of node caused by listening time.

\subsection{Improved S-MAC}

Data transmission from source node A to node $\mathrm{E}$ will be accomplished in one dormancy cycle with 4 hops in total. To compare with above two protocols, adopt the same assumption condition for analyzing the improved S-MAC, which is to analyze the delay of MAC protocol at light load and there is only one group transmitted in network. In this way, there is no queuing delay and back off delay. Further assume that transmission delay and processing delay can be ignored. Under this situation, we only consider about listening delay tcs, transmission delay ttx and dormancy delay ts,n, as shown in Figure 11.

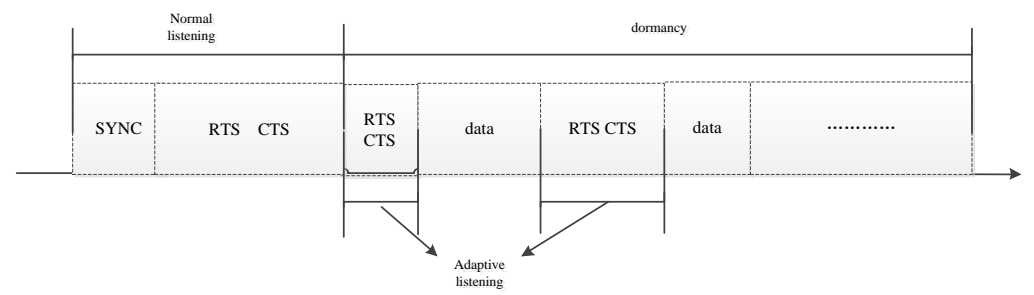

\section{Figure 10. Normal Listening and Adaptive Listening In}

With this assumption, in the improved S-MAC protocol, the transmission delay of data grouping through $\mathrm{n}$ nodes is:

$$
D(N)=t_{s, 1}+t_{c s, 1}+t_{c s, 2}+t_{c s, 3}+t_{t x}+t_{c s, 4}+\mathrm{t}_{\mathrm{s}, 5}+\cdots+\mathrm{t}_{\mathrm{cs}, \mathrm{N}-1}+\mathrm{t}_{\mathrm{tx}}+\mathrm{t}_{\mathrm{cs}, \mathrm{N}}+\mathrm{t}_{\mathrm{tx}}
$$

The relationship between one frame length $\mathrm{Tf}$ and several delays can be attained through Figure 12:

$$
T f=t_{c s, \mathrm{~J} 1}+t_{t x}+t_{c s, \mathrm{n}+1}+t_{t x}+t_{c s, \mathrm{n}+2}+\mathrm{t}_{t x}+\mathrm{t}_{\mathrm{cs}, \mathrm{n}+3}+\mathrm{t}_{\mathrm{tx}}+\mathrm{t}_{\mathrm{s}, \mathrm{I} 1+4}
$$

Simplify equation (14) with equation (15), the delay is:

$$
D(N)=t_{s, 1}+(N / 4-1) T_{f}+t_{c s, \mathrm{~N}-3}+t_{c s, \mathrm{~N}-2}+t_{c s, N-1}+t_{c s, \mathrm{~N}}+4 \mathrm{t}_{t x}
$$

Similarly, the dormancy delay of the first node still adopts its average value $\mathrm{Tf} / 2$; therefore, the average delay through $\mathrm{N}$ nodes is:

$$
E[D(N)]=T_{f} / 2+(N / 4-1) T_{f}+4 t_{c s}+4 t_{t x}
$$

After simplification:

$$
E[D(N)]=N T_{f} / 4-T_{f} / 2+4 t_{c s}+4 t_{t x}
$$

It can be seen from the above average delay that in adaptive S-MAC, delay keeps an increasing trend with the increase of nodes. However, it slows down in the rising of improved S-MAC; the slope is Tf/4, which is only $1 / 4$ of basic S-MAC protocol and $1 / 2$ of adaptive S-MAC. It can be seen that the improved S-MAC protocol also increased greatly in delay even compared with adaptive S-MAC. 


\section{Network Simulation}

\subsection{End to End Delay}

First, we test the performance of each protocol at delay. The tested network environment is a light load environment. In the whole network, there is only one data group transmitted in network and the group does not need to be fragmented. In three different versions of protocol testing, all of them adopt frame length with 10 seconds, $10 \%$ work cycle and transmission distance of node signal is 1.5 times of node distance. We have tested ten times of transmission time test from the 1st hop to the 10th hop for each version of M-MAC protocol. The transmission time starts from all nodes entering dormancy-work states to designated destination node connecting to data grouping.

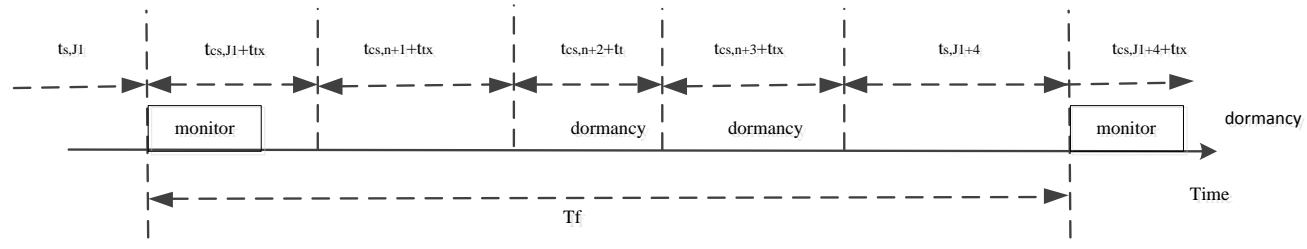

Figure 11. A sleep cycle for Four Adaptive Transmission

Figure 12 displays the time consumed by basic S-MAC protocol, adaptive S-MAC protocol and Improved S-MAC protocol for transmitting one group in different hops test. It can be seen from the Figure that the end to end delay of these three increases with the increase of hops, only the slopes are different. In the test with only one hop, the consumed time for these three is the same. In the test with two hops, the delay of adaptive S-MAC and improved S-MAC is the same. In the following test results, adaptive S-MAC and improved S-MAC demonstrate own advantages gradually. The delay of adaptive S-MAC is a little more than a half of basic S-MAC while the result of improved S-MAC is only a little more than a half of adaptive S-MAC. It can be seen from this result that the adaptive S-MAC with adoption of adaptive listening technology decreases delay greatly when compared with original basic S-MAC, while the improved S-MAC reaches the purpose of decreasing end to end delay on adaptive S-MAC protocol in an further way and builds up good foundation for improving system response time.

\subsection{System Energy Consumption}

We mainly adopt following network environment parameters for testing the performance of each protocol in energy consumption:

1. Frame length: 10 seconds

2. Work cycle: $10 \%$ frame length

3. Node signal transmission distance: 1.5 times of node distance

4. Groups of each test transmission data: 5

5. Data grouping transmission hops: 10 hops

It can be seen from Figure 13 that basic S-MAC consumes more energy than the other two versions of S-MAC, while the energy consumption of the other two protocols is basically the same. For S-MAC protocol adopting adaptive listening technology, it increases the listening time of participating adaptive transmission nodes, but it decreases end to end delay and the listening time of all nodes in the whole network decreases, therefore, it is reasonable for less energy consumption. The improved S-MAC is the further strengthening of adaptive listening technology and there are a lot of nodes participating in adaptive listening, but our improve decreases partial invalid adaptive listening, moreover, it also decreases some delay when compared with adaptive S-MAC. 
Therefore, as shown in the test results of Figure 13, the energy consumptions of both are nearly the same.

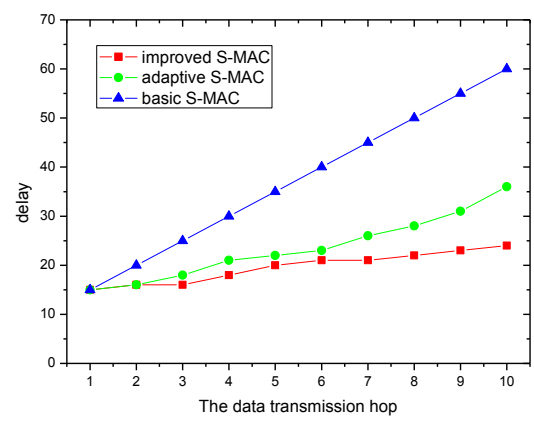

Figure 12. Network Latency

\subsection{Network Throughput}

Network throughput presents the ability of network in transmitting data and is an important index of network performance, therefore, we also make test for the network throughput of each protocol. We mainly adopt the following network environment parameters during test:

1. Frame length: 10 seconds

2. Work cycle: $10 \%$ frame length

3. Node signal transmission distance: 1.5 times of node distance

4. Groups of each test transmission data: 5

5. Data grouping transmission hops: 10 hops

It can be seen from Figure 14 that the throughputs of these three protocols are not big and the biggest one is $4.5 \mathrm{byte} / \mathrm{s}$, but the test result of Wei Ye and others is about 40byte/s, which is mainly because that the frame we adopted is ten seconds while they adopted one second. The difference exists but it will not affect our analysis for these three protocols.

In view of the test results of network throughput, the network throughput of three protocols decreases with the decrease of network load. Adaptive S-MAC transmits data group by two times within one dormancy cycle, therefore, the whole system transmission speed is faster than basic S-MAC and then the system throughput has been improved obviously. The improved S-MAC improves transmission speed in a further way, whose throughput has been improved obviously compared with original S-MAC network.

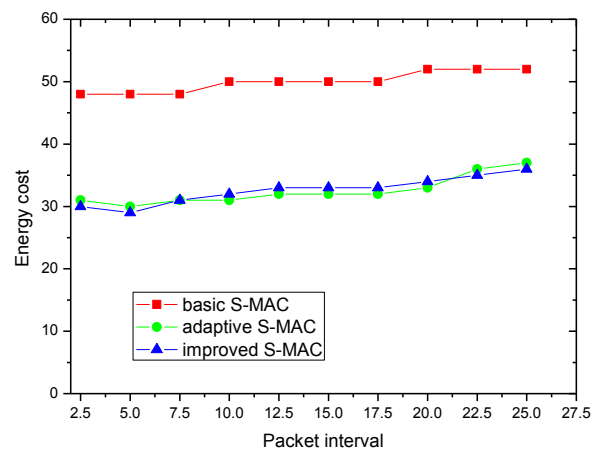

Figure 13. The System Energy Consumption 


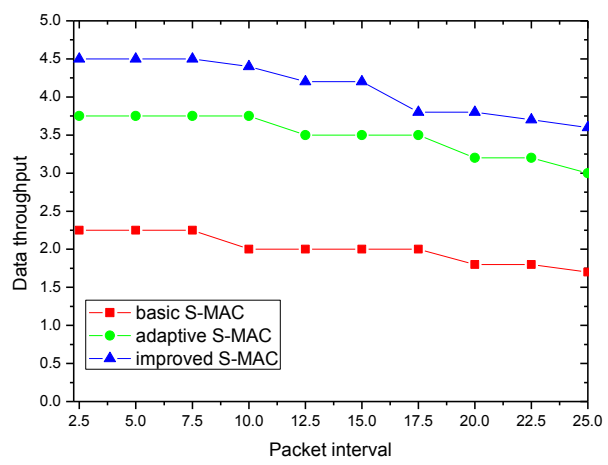

Figure 14. Network Throughput

\section{Conclusion}

As a hot topic in wireless sensor network focusing on energy, MAC technology which plays an important role in it will definitely attract more and more attention. On the basis of studying the famous S-MAC protocol, we strengthen the application of adaptive listening technology in S-MAC. When the nodes have no data to process, they are in dormancy state for most of the time, while when the data is coming, they become active quickly and participate in data transmission actively. The nodes which can feel the transmission is just around will participate in ad aptive transmission as much as possible and make continuous adaptive listening within one dormancy period. In this way, realize the purpose of deceasing end to end delay greatly on the basis of not increasing additional energy consumption. For applications which are sensitive to system response time, the improved S-MAC is with greater application value.

\section{Acknowledgments}

The work was supported by Scientific Support Plan Projects in Langfang City(No.2016011012); Teachers' Scientific Research Fund of China Earthquake Administration (No.20140104).

\section{References}

[1] S. Sung, H. Kang, E. Kim and K. Kim, "Energy Consumptlon Analysls of S-MAC Protocol in SingleHop Wireless Sensor Networks", Proceedings of Asia-Pacific Conference on Communications, APCC, Busan, Korea, (2006).

[2] D. Saha, M. R. Yousuf and M. A. Matin, "Energy Efficient Scheduling Algorithm for S-MAC Protocol in Wireless Sensor Network", International Journal of Wireless \& Mobile Computing, vol. 3, no. 6, (2011), pp. 4350-4353.

[3] S. Toklu and O. A. Erdem, "BSC-MAC: Energy efficiency in wireless sensor networks with base station control", Journal of Computer Networks, vol. 59, no. 3, (2014), pp. 91-100.

[4] S. Ray, I. Demirkol and W. Heinzelman, "ADV-MAC: Advertisement-based MAC Protocol for wireless sensor networks", Proceedings of the 5th International Conference on Mobile Ad-Hoc and Sensor Networks, Fujian Province, China, (2009).

[5] T. S. Lee, Y. Yang, K. J. Shin and M. S. Park, "An Energy-Efficient Uni-scheduling Based on S-MAC in Wireless Sensor Network", Proceedings of the 1st International Conference on High Performance Computing and Communcations, Sorrento, Italy, (2005).

[6] W. Lee, M. Nguyen, A. Verma and H. S. Lee, "Schedule unifying algorithm extending network lifetime in S-MAC-based wireless sensor networks", IEEE Transactions on Wireless Communications, vol. 8, no. 9, (2009), pp. 4375-4379.

[7] S. Du, A. K. Saha and D. B. Johnson, "RMAC: A Routing-Enhanced Duty-Cycle MAC Protocol for Wireless Sensor Networks", Proceedings of the 26th IEEE International Conference on Computer Communications, Anchorage, AK, United states, (2007). 
[8] S. Ghosh and P. Veeraraghavan, "Energy efficient medium access control with single sleep schedule for wireless sensor networks", Proceedings of IEEE International Conference on Telecommunications and Malaysia International Conference on Communications, Penang, Malaysia, (2007).

[9] Y. Zhang, C. He and L. Jiang, "Modeling the S-MAC Protocol in Single-Hop Wireless Sensor Networks", Proceedings of IEEE International Conference on Communications, Beijing, China, (2008).

[10] L. L. Gao, "A energy consumption improvements of S-MAC in WSN", Proceedings of International Conference on Internet Technology and Applications, Wuhan, China (2011).

[11] T. Chiras, M. Paterakis and P. Koutsakis, "Improved medium access control for wireless sensor networks - a study on the S-MAC protocol", Proceedings of the 14th IEEE Workshop on Local and Metropolitan Area Networks, Chania, Greece, (2005).

[12] H. S. Aghdasi and M. Abbaspour, "ET-MAC: An Energy-Efficient and High Throughput MAC Protocol for Wireless Sensor Networks", Proceedings of the 6th Annual Communication Networks and Services Research Conference, Halifax, NS, Canada, (2008).

[13] Z. Ye, H. Chen and L. Jiang, "Energy and QoS trade-off analysis of S-MAC protocol in wireless sensor networks", Atmospheric Chemistry \& Physics, vol. 8, no. 4, (2008), pp. 76-79.

[14] I. Ammar, I. Awan and G. Min, "An Improved S-MAC Protocol Based on Parallel Transmission for Wireless Sensor Networks", Proceedings of 13th International Conference on Network-Based Information Systems, Gifu, Japan, (2010).

[15] D. Saha and M. A. Matin, "A new Uni-Scheduling algorithm in S-MAC based wireless sensor networks", Proceedings of International Conference on Wireless Communications and Signal Processing, Nanjing, China, (2011).

[16] A. N. A. Pantazis, A. Pantazis, S. A. Nikolidakis and D. D. Vergados, "A performance evaluation of SMAC protocol in combination with energy efficient protocols for Wireless Sensor Networks", Proceedings of the 18th International Conference on Telecommunications, Ayia Napa, Cyprus, (2011).

[17] F. Hamady, M. Sabra, Z. Sabra, A. Kayssi, A. Chehab and M.Mansour, "Enhancement of the S-MAC protocol for wireless sensor networks", Proceedings of International Conference on Energy Aware Computing, Cairo, Egypt, (2010).

[18] S.Y. Wang, L.M. Jia, Y.M. He. Intelligent Irrigation System Based On S3C6410. Revista de la Facultad de Ingeniería, vol. 31, no. 6, 2016, pp. 219-232.

[19] T. Zhang and K. I. Kim, "Performance Study for S-MAC in Wireless Sensor Notworks", Journal of Information \& Communication Convergence Engineering, vol. 8, (2010), pp. 122-128.

[20] S. Nguyen-Xuan, S. Oh and S. An, "EE-MAC: Energy efficient-medium access control for periodic applications in border surveillance wireless sensor networks", Proceedings of the 16th International Conference on Advanced Communication Technology, PyeongChang, Korea, (2014).

[21] P. T. Kalaivaani and A. Rajeswari, "An Energy Efficient Analysis of S-MAC and H-MAC Protocols for Wireless Sensor Networks”, International Journal of Computer Networks \& Communications, vol. 5, no. 2, (2013), pp. 83-94.

[22] R. Alageswaran, S. Prasakthi and V. Vaithiyanathan, "Comparative Study of QoS and Non-QoS MAC Protocols of Wireless Sensor Networks", International Journal of Applied Engineering Research, vol. 8, no. 20, (2013), pp. 2711-2716.

[23] L. G. J. H. Zheng, "Research on Evaluation Method of Hierarchical Network Security Threat", Revista de la Facultad de Ingeniería, vol. 31, no. 5, (2016), pp. 49-58.

[24] H. C. Jing, "The Study on the Impact of Data Storage from Accounting Information Processing Procedure", International Journal of Database Theory and Application, vol. 8, no. 3, (2015), pp. 323-332.

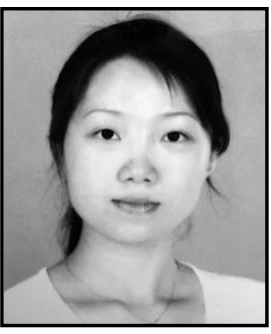

\section{Authors}

Yan Shi, she was born in 1981. In 2008, she received her M.E. degree in software engineering at Beijing University of Posts and Telecommunications. She received her B.E. degree in Information Engineering at Chengdu University of Technology. Her major fields of study are wireless communication. 
International Journal of Future Generation Communication and Networking Vol. 9, No. 12 (2016) 